doi:10.1017/S1041610219001406

\section{An empirical validation of neuropsychiatric symptoms and agitation profiles in dementia using data from an online symptom tracking tool}

The internet offers a powerful means of gathering information about the lived experience of dementia, especially with patients and caregivers increasingly using online tools or apps for symptom management. A recent study by our group (Rockwood et al., 2019) employed such a tool to explore agitation from usercompleted online reports $(n=2,577)$ on a dementia symptom tracking website, www.dementiaguide.com. Website users ( $97 \%$ of whom are caregivers) selected symptoms to track to better understand the evolution of dementia, and/or assess response to treatment in the person for whom they care. The study compared the proportion of people with neuropsychiatric symptoms and agitation, and the number of such symptoms, across groups with mild cognitive impairment (MCI), and mild, moderate, and severe dementia. Important to readers of International Psychogeriatrics, agitation was operationalized using terms consistent with the International Psychogeriatric Association (IPA) consensus definition (Cummings et al., 2015) and the Neuropsychiatric Inventory-Questionnaire (NPI-Q) agitation/aggression domain (Cummings 1997). This facilitated comparison between the agitation profiles found on our website and those described in other settings. We found that the proportion with neuropsychiatric symptoms and agitation symptoms increased with increasing cognitive impairment, as did the number of neuropsychiatric symptoms, including symptoms associated with agitation as per the IPA consensus definition (Cummings et al., 2015).

Of broader interest is the notable similarity between our estimates of the frequency of neuropsychiatric symptoms generally and agitation symptoms specifically and those from clinically adjudicated reports that are acquired using traditional data collection approaches. For example, Siafarikas et al. (2018), using data on 2,141 patients in the Norwegian national registry of memory clinics, investigated neuropsychiatric symptoms in relation to dementia. As part of our validation study, we compared trends in the two reports. We found that estimates of the proportions of people with at least one neuropsychiatric symptom were consistent (Table 1). Similar comparability was observed with regard to agitation. Further support for the validity of the IPA consensus and of using online data comes from a study by Peters et al. (2006) that employed data from A Canadian Cohort Study of Cognitive Impairment and Related Dementias (ACCORD) (Feldman et al., 2003) to characterize neuropsychiatric symptoms $(n=769)$ (Table 1). Despite differences in selection and reporting procedures between the studies, it appears that once a sample of people in whom a high proportion have dementia is assembled, comparable associations between neuropsychiatric symptoms and estimates can be observed. The similarity in the trends observed in our online database and estimates from clinically adjudicated reports suggests both validity of the IPA definition and the merit in exploring the internet as a means to capture the lived experience of people with dementia.

\section{Statement of Interests}

In addition to academic and hospital appointments, $\mathrm{KR}$ is president and chief science officer of DGI Clinical, which in the last 5 years had contracts with pharma and device manufacturers (Baxter, Baxalta, Biogen, Shire, Hollister, Nutricia, Roche, and

Table 1. Proportions of people with neuropsychiatric symptoms and agitation symptoms in samples with a high proportion of people living with cognitive impairment

\begin{tabular}{|c|c|c|c|c|c|c|}
\hline & \multicolumn{3}{|c|}{$\begin{array}{l}\text { PROPORTION OF PEOPLE WITH AT LEAST } \\
\text { ONE NEUROPSYCHIATRIC SYMPTOMS }\end{array}$} & \multicolumn{3}{|c|}{$\begin{array}{c}\text { PROPORTION OF PEOPLE WITH } \\
\text { AGITATION }\end{array}$} \\
\hline & $A L L S T A G E S$ & $M C I$ & DEMENTIA ${ }^{*}$ & ALL STAGES & $M C I$ & DEMENTIA \\
\hline Rockwood et al. (2019) & $83 \%$ & $76 \%$ & $97 \%$ & - & $19 \%$ & $40 \%$ \\
\hline Siafarikas et al. (2018) & $87.2 \%$ & $79.5 \%$ & $91.2 \%$ & - & $23 \%$ & $35 \%$ \\
\hline Peters et al. (2006) & - & $74 \%{ }^{\dagger}$ & $89 \%$ & - & $23 \% \dagger$ & $36 \%$ \\
\hline
\end{tabular}

*For Rockwood et al. (2019) this figure reflects the proportion in those with severe dementia, and for Siafarikas et al. (2018) this figure reflects the proportion in those with $\mathrm{AD}$.

tPeters et al. This figure reflects the proportion in those that were deemed cognitively impaired-not demented (CIND). 
Otsuka) on individualized outcome measurement. In 2017, he attended an advisory board meeting with Lundbeck. Otherwise, any personal fees are for invited guest lectures, rounds and academic symposia, received directly from event organizers, for presentations on frailty. $\mathrm{He}$ is the associate director of the Canadian Consortium on Neurodegeneration in Aging, which is funded by the Canadian Institutes of Health Research (CNA-137794), with additional funding from the Alzheimer Society of Canada and several other charities, as well as from Pfizer Canada and Sanofi Canada (in phase 1, 2014-2019). He receives career support from the Dalhousie Medical Research Foundation as the Kathryn Allen Weldon Professor of Alzheimer Research and research support through grants from the Canadian Institutes of Health Research, the Canadian Frailty Network, the Nova Scotia Health Research Foundation, the Nova Scotia Health Authority Research Fund, and the Fountain Family Innovation Fund of the QEII Health Science Centre Foundation. JS and LM are employees of DGI Clinical Inc.

\section{References}

Cummings, J. et al. (2015). Agitation in cognitive disorders: International Psychogeriatric Association provisional consensus clinical and research definition. International Psychogeriatrics, 27, 7-17. doi: 10.1017/ S1041610214001963.

Cummings, J. L. (1997). The Neuropsychiatric Inventory Assessing psychopathology in dementia patients. Neurology, 48, 10S-6S. doi: 10.1212/wnl.48.5_suppl_6.10s.
Feldman, H. et al. (2003). A Canadian cohort study of cognitive impairment and related dementias (ACCORD): study methods and baseline results. Neuroepidemiology, 22, 265-274. doi: 10.1159/000071189.

Peters, K. R. et al. (2006). Characterizing neuropsychiatric symptoms in subjects referred to dementia clinics. Neurology, 66, 523-528. doi: 10.1212/01.wnl.0000198255 .84842 .06 .

Rockwood, K. et al. (2019). The symptoms targeted for monitoring in a Web-based tracking tool by caregivers of people with dementia and agitation: Cross-sectional study. Fournal of Medical Internet Research, 21, e13360. doi: 10 $.2196 / 13360$.

Siafarikas, N., Selbaek, G., Fladby, T., Benth, J. Š., Auning, E. and Aarsland, D. (2018). Frequency and subgroups of neuropsychiatric symptoms in mild cognitive impairment and different stages of dementia in Alzheimer's disease. International Psychogeriatrics, 30, 103-113. doi: 10 $.1017 /$ S1041610217001879.

KenNeth Rockwood, ${ }^{1,2}$ (i) Justin STanley ${ }^{2}$ and LISA MCGARRIGLE ${ }^{2}$

${ }^{1}$ Division of Geriatric Medicine, Department of Medicine, Dalhousie University and Nova Scotia Health Authority, 5955 Veterans' Memorial Lane, Halifax NS B3H 2E1, Canada

${ }^{2}$ DGI Clinical Inc., 1730 Market St, Halifax, NS B3J 3N9, Canada

Correspondence should be addressed to: Kenneth Rockwood, Centre for Health Care of the Elderly, Nova Scotia Health Authority, 1421-5955 Veterans' Memorial Lane, Halifax, Nova Scotia, Canada, B3H 2E9. Phone: (902) 473-8687. Email: kenneth.rockwood@dal.ca. 